\title{
Ion characteristics of laser-produced plasma using a pair of collinear femtosecond laser pulses
}

\author{
Z. Zhang, P. A. Van Rompay, and P. P. Pronko ${ }^{\text {a) }}$ \\ Center for Ultrafast Optical Science and Department of Electrical Engineering and Computer Science, \\ University of Michigan, Ann Arbor, Michigan 48109-2099
}

(Received 25 January 2002; accepted 15 May 2003)

\begin{abstract}
Femtosecond laser-pulse absorption is studied in silicon ablation plasmas by means of a pair of identical $10^{16} \mathrm{~W} / \mathrm{cm}^{2}$ collinear pulses separated on a picosecond time scale. The second laser-pulse modifies ionic characteristics of the preformed plasma, such as ion yield, ion energy, and average charge state. Resonance absorption is demonstrated to be the dominant mechanism by comparing results of $s$ and $p$ polarization. It is shown that maximum effects occur when a well defined critical density surface of the initial plasma forms together with an optimum density gradient scale length of $k L=1.5$. The optimal enhancement of ion yield, which occurs at 5 ps delay, is a factor of 2 greater than that produced by a single pulse with twice the energy of each individual double pulse. Applications are identified in regard to cluster beam formation and plasma isotope enrichment in ultrafast ablation plumes. (c) 2003 American Institute of Physics. [DOI: 10.1063/1.1592616]
\end{abstract}

Absorption mechanisms for femtosecond laser pulses in preformed plasmas have been previously studied using optical absorption and/or x-ray excitation techniques. ${ }^{1-7}$ It has been shown in these earlier works that collisional and resonance absorption are the two dominant processes occurring in plasmas that start out with moderate to steep density gradient scale lengths. Although ionic yield measurements with double pulses have been done in dilute gases ${ }^{8}$ little or no published literature appears concerning such ionic measurements for high intensity short scale length plasmas from solid surfaces. This is especially true in regard to charge state and energy dependence. In this letter, we report an experimental investigation into the enhancement of ionic properties in the plasma portion of ablation plumes from a silicon target resulting from two identical 110 fs laser pulses separated by 0-40 ps. Time-resolved measurements were made of ion flux, ion energy, and average charge state to verify that the ionic character of a plasma, initially formed by a single pulse, can be subsequently modified, in the far field, by the pumping action of a secondary time-delayed pulse and that location of the plasma critical density surface and the density scale length of the expanding plasma can be usefully exploited.

The laser source was a Ti:sapphire chirped-pulse amplification laser system operated at $10 \mathrm{~Hz}$. It provided $110 \mathrm{fs}, 36$ $\mathrm{mJ}$ laser pulses at a wavelength of $780 \mathrm{~nm}$. For this experiment, a single laser pulse was split into two identical pulses with a variable time delay using a Michelson interferometer. The resolution of the time delay was 0.2 ps. Both pulses were either $p$ or $s$ polarized and focused collinearly on a silicon target (in $5 \times 10^{-8}$ Torr vacuum) using a $750 \mathrm{~mm}$ focal-length lens at an incident angle was $45^{\circ}$. Time-of-flight (TOF) ion signals are measured using a curved-sector, electrostatic energy analyzer to obtain energy distributions as a function of charge state. Alternatively, the ion flux can be allowed to proceed undeflected into a straight-through port of the analyzer to obtain an undifferentiated ion-flux density

a)Electronic mail: pronko@eecs.umich.edu and TOF average ion energy. For additional details see Ref. 9.

Figure 1 shows the total density of ions (ions/steradian) and average ion energy in the ablation plume produced by a pair of pulses, as a function of time delay. The data points at zero delay are the result of a single pulse (2 $\times 10^{16} \mathrm{~W} / \mathrm{cm}^{2}$ ) with twice the energy as that contained in each of the individual double pulses $\left(1 \times 10^{16} \mathrm{~W} / \mathrm{cm}^{2}\right)$. Two effects are observed in the TOF data shown in Fig. 1. First, at 5 ps delay, where the maximum effect of the second pulse occurs, the ion yield is twice as high as that produced by a single full-energy pulse. Second, the ion average kinetic energy increases to a maximum of $5.3 \mathrm{keV}$, using double pulses separated by $5 \mathrm{ps}$, compared with $3.2 \mathrm{keV}$ using a single pulse of twice the energy. This most likely results from the bipolar electrostatic field, responsible for ion acceleration, being reinforced by additional electrons from the second laser pulse so that ions experience a stronger accelerating field. The enhancement of both ion yield and ion energy confirms that the second pulse does not produce an independent ablation event but rather couples into the plasma generated by

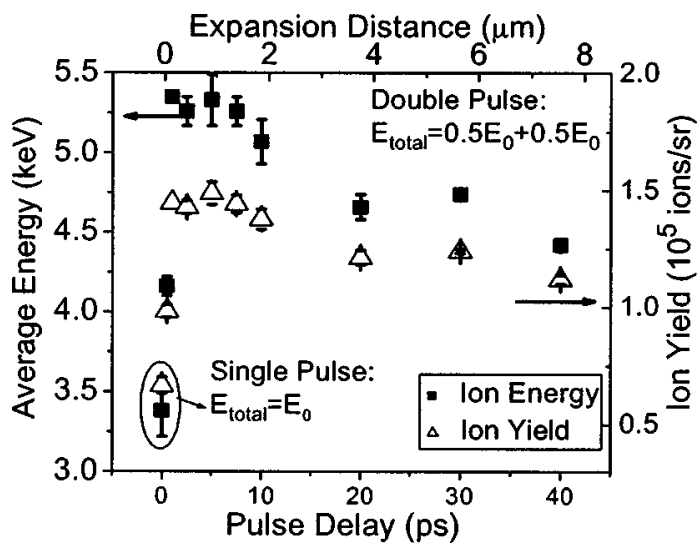

FIG. 1. Average ion yield and energy as a function of time delay between two identical $120 \mathrm{fs}$ pulses $\left(1 \times 10^{16} \mathrm{~W} / \mathrm{cm}^{2}\right)$ on silicon. At zero delay the single pulse has an intensity of $2 \times 10^{16} \mathrm{~W} / \mathrm{cm}^{2}$. Expansion distance is based on measured average ion velocity of $1.9 \times 10^{7} \mathrm{~cm} / \mathrm{s}$. 


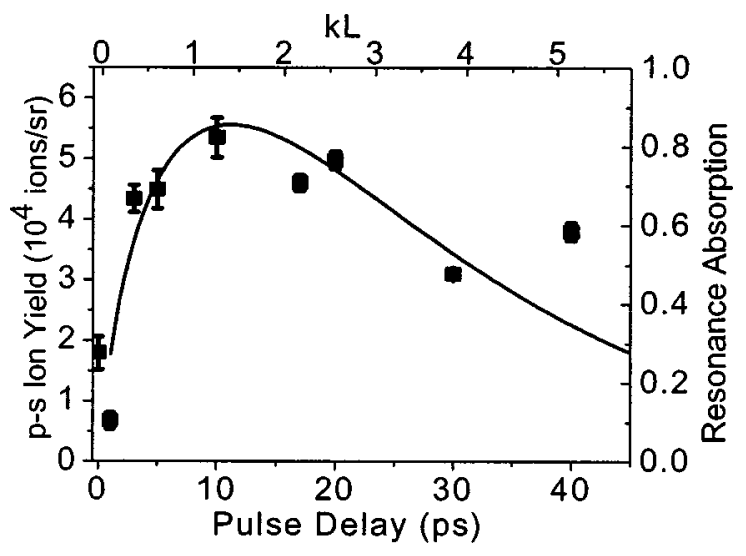

FIG. 2. Subtracted difference in ion yield for $p$ - and $s$-polarized pulses as a function of time delay. Solid line is the fit to experimental ion yield difference data by calculating the resonance absorption function in terms of scale length.

the first pulse. In Fig. 1, the maximum enhancement at 5 ps delay implies that this time interval is required to allow for sufficient expansion of the initially overdense plasma to develop suitable conditions to absorb the second pulse. These conditions include an appropriate density gradient with a properly positioned critical surface, whereupon it can again become an efficient optical absorber. Critical density (1.7 $\times 10^{21} \mathrm{~cm}^{-2}$ ) is defined here in the usual way as $n_{c}$ $=m \omega^{2} / 4 \pi e^{2}$, where $n_{c}$ is the electron density, $m$ is the electron mass, $\omega$ is the laser frequency, and $e$ is the electronic charge. Since in Fig. 1 the incident pulses are $p$ polarized, both collisional and resonance absorption processes could take place. Collisional absorption normally occurs in the outer corona of the plasma and can be very efficient over large portions of the under dense regions of the plasma. It is known to increase with increasing scale length. ${ }^{10}$ Resonance absorption occurs near the critical density surface when part of the light wave tunnels into the overdense region to excite electrons into oscillation along the density gradient, thereby producing resonant plasma oscillation..$^{10}$ The energy of such a resonant wave is then damped into the plasma by either collisional or non-collisional process. Given the differing characteristics of these two processes, the plasma density gradients required to optimize each of the mechanisms will not be the same nor will their functional dependence on the delay time be the same. In order to better understand these mechanisms, experiments were performed with both $s$ - and $p$-polarized pulses to isolate the effects of collisional and resonance absorption. The subtracted difference in ion yield for the two cases was examined and the results presented in Fig. 2. In obtaining those results, an s-polarized absorption contribution was observed to briefly occur around 1 ps delay and then to rapidly disappear thereafter. This is contrasted with the broad absorption occurring in Fig. 1 for $p$-polarized pulses extending over a range of delays out to $40 \mathrm{ps}$. The increase in ionization yield obtained using oblique incidence $p$-polarized light will be proportional to the energy absorbed from the second pulse. The absorbed energy flux, for a linear density gradient, is given ${ }^{10}$ as $F_{\mathrm{abs}}=(c / 16 \pi) E_{\mathrm{fs}}^{2} \Phi^{2}(\tau)$ where $c$ is the velocity of light, $L$ is the density gradient scale length, and $E_{\mathrm{fs}}$ is the laser electric field in free space, a component of which is responsible for driving the resonance

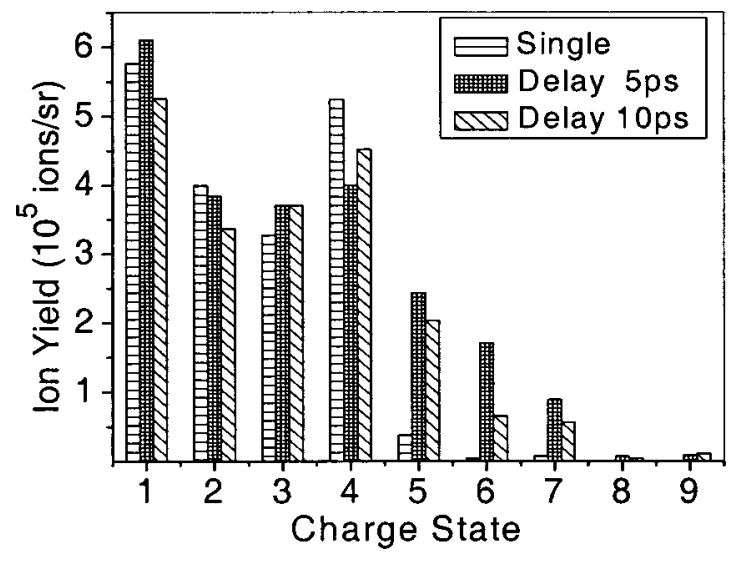

FIG. 3. Discrete charge-state distributions as a function of time delay for 0 , 5 , and $10 \mathrm{ps}$, respectively. Individual pulses of intensity $1 \times 10^{16} \mathrm{~W} / \mathrm{cm}^{2}$.

electron oscillation along the density gradient. That component is controlled by the term $\Phi(\tau) \cong 2.3 \tau \exp \left(-2 \tau^{3} / 3\right)$ where $\tau=(k L)^{1 / 3} \sin \theta$. The parameter $k$ is the laser optical wave number and $\theta$ is the angle of incidence of the laser pulse on the target. For a fixed angle of incidence, as is the case in the present work, $\Phi(\tau)$ becomes a function of plasma density scale length only. In our experiment the scale length of the expanding plasma will be determined by the delay time between the first and second pulse. Therefore, under resonance absorption conditions, our ion yield should be a well defined function of delay time and should have the functional form of $\Phi^{2}(\tau)$. In Fig. 2, the calculated values of $\Phi^{2}(\tau)$, are plotted as a function of scale length $(k L)$ and compared to our experimental $p$ minus $s$ yield data as a function of delay time. The calculated absorption curve fits exceptionally well with the enhanced ion yield data. In making this comparison, the calculated absorption curve gives a $k L$ value of 1.5 for the optimum delay time of $5 \mathrm{ps}$. These values are in good agreement with experiments of others. ${ }^{2,4}$ They also are in reasonable agreement with 5 ps gradient scale lengths from our own simulation results ${ }^{11}$ and that of others. $^{12}$

Figure 3 shows the distribution of charge states for three different delays corresponding to the conditions of Figs. 1 and 2 . The average ion charge in the plume for the zero delay double pulse is 2.5 . For the case with a 5 ps time delay, it increases to 3.1, and for a $10 \mathrm{ps}$ delay, it becomes 3.0. It is seen in Fig. 3 that absorption of the second pulse results in a significant increase of the higher charge-state ions $(+5$ to +7 ) while having modest or no effect on the number of low charge-state ions. The collisional-radiative equilibrium plasma model ${ }^{13}$ is often used to describe such plasma ionization dynamics. This model normally shows an increase in the percentage of higher charge-state ions at the expense of lower charge states as the plasma temperature increases. However, in this experiment, the increase of average ionic charge does not occur at the expense of the low charge-state ions but rather by only generating a larger number of high charge-state ions. Under resonance absorption it can be expected that the additional contribution to charge states will be associated with absorption of the second pulse predominantly in the leading edge of the over dense portion of the expanding plasma (near the critical density surface). This is, however, where the high-velocity high-charge-state ions nor- 


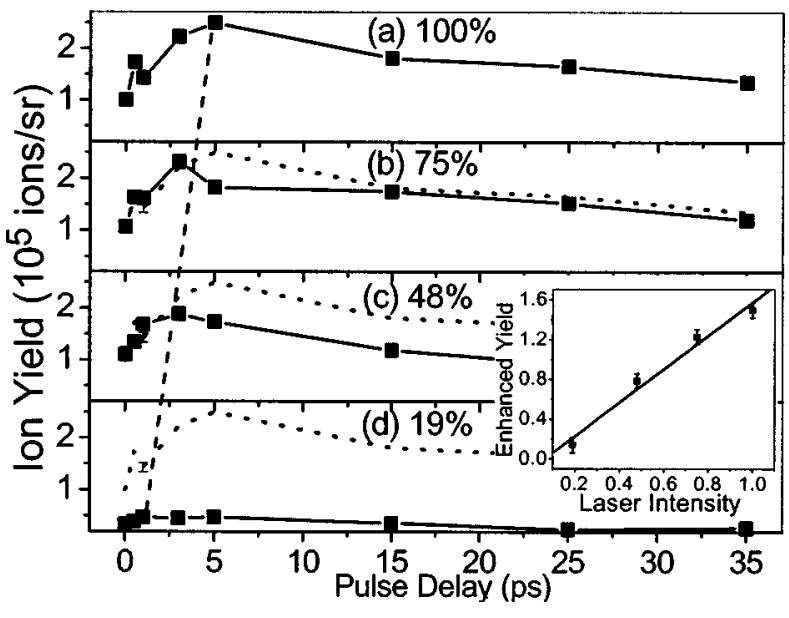

FIG. 4. Ion yields as a function of time delay between two identical pulses for a silicon ablation plume. The laser intensity is represented as a percentage of the maximum intensity $\left(1 \times 10^{16} \mathrm{~W} / \mathrm{cm}^{2}\right)$ and is shown on each subfigure. The diagonal dashed line is drawn through the peak of each delay curve showing the delay time for maximum enhancement. Maximum enhanced ion yields in the unit of $10^{5}$ ions/sr, as a function of laser intensity in the unit of $10^{16} \mathrm{~W} / \mathrm{cm}^{2}$, are shown in the inset.

mally reside, resulting in their being affected most. These results further confirm therefore the conclusions from Fig. 2 attributing the absorption of the second pulse to a resonance process.

Ion yields as a function of pulse separation are plotted in Figs. 4(a), 4(b), 4(c), and 4(d) using 100\%, 75\%, 48\%, and $19 \%$ of the maximum laser intensity per pulse (1 $\times 10^{16} \mathrm{~W} / \mathrm{cm}^{2}$ ), respectively. In comparing these data, we observe that the enhancement of ion yield (shown) and ion average energy (not shown) systematically decrease with laser intensity. It is also observed that the time to achieve maximum absorption is lower with decreasing laser pulse intensity. Based on a resonance absorption process, the reduction in ion yield can be related to the fraction of energy absorbed from the second pulse as a function of laser intensity. As seen in the previously stated expression for absorbed flux, $F_{\mathrm{abs}} \alpha E_{\mathrm{fs}}^{2} \alpha I$, the laser intensity. The inset of Fig. 4 shows the ion yield enhancement as a function of laser intensity taken from Figs. 4(a), 4(b), 4(c), and 4(d). It is seen there that at the expected linear relation is well represented. The reduction in delay time to achieve maximum absorption is a less obvious result; however, under resonance absorption it will occur when the initially expanding plasma achieves a well-defined critical density surface simultaneous with establishment of an optimum gradient scale length. For example, at $1 \times 10^{16} \mathrm{~W} / \mathrm{cm}^{2}$, simulations ${ }^{11}$ show that a time span of about 5 ps is needed for the critical surface to clearly emerge from the initially overdense compact density profiles. Prior to that the gradients are so steep that the density contours are simply not well separated. At lower intensities, these same simulations show that it takes less time for such conditions to become established. Continued work on these simulations is underway to further understand the processes involved. Additional effects may be associated with the results of Fig. 4 in that, at the higher values of $I \lambda^{2}$, a steepening of the density profile, due to radiation pressure ${ }^{14}$ and pondermotive effects, ${ }^{15}$ will occur and could affect the time to maximum absorption. Such processes will be less pronounced at the lower intensities.

In summary, double pulses separated on a picosecond time scale effectively increase ion yield, ion kinetic energy, and average charge in the plasma compared with those produced at zero delay. Peak enhancement occurs when the plasma develops an optimum density gradient, at a specific angle of incidence, along with a well-defined critical density surface, such that they are collectively consistent with the requirements of maximum resonance absorption. Similar optimum delay and enhancement effects were observed in previous in laser-produced $\mathrm{x}$-ray experiments. ${ }^{6,7}$ Experimentally these time-correlated enhancements are an interesting and potentially useful method for studying and manipulating plasma expansion processes. For example, and as we have recently shown, a secondary time-delayed pulse can be used to reinforce such effects as isotope separation and cluster beam formation in ultrafast ablation plumes. ${ }^{16}$ An operational approach can be envisioned, for such technological applications, where, by taking advantage of profile steepening, multiply-sequenced pulses could be used to maximize the ionic component of the plume or the internal energy of the initially expanding zone of the plasma. One can anticipate such conditions being useful for applications of ultrafast ablation plumes in materials science and ion physics.

This work was supported by the Chemical Sciences, Geosciences and Biosciences Division, Office of Basic Energy Sciences, U.S. Department of Energy.

${ }^{1}$ J. C. Kieffer, J. P. Matte, S. Belair, M. Chaker, P. Audebert, H. Pepin, P. Maine, D. Strickland, P. Bado, and G. Mourou, IEEE J. Quantum Electron. 25, 2640 (1989).

${ }^{2}$ U. Teubner, J. Bergmann, B. van Wonterghem, F. P. Schafer, and R. Sauerbrey, Phys. Rev. Lett. 70, 794 (1993).

${ }^{3}$ R. Fedosejevs, R. Ottmann, R. Sigel, G. Kühnle, S. Szatmari, and F. P. Schäfer, Phys. Rev. Lett. 64, 1250 (1990).

${ }^{4}$ C. Y. Cote, J. C. Kieffer, and O. Peyrusse, Phys. Rev. E 56, 992 (1997).

${ }^{5}$ O. L. Landen, D. G. Sterarns, and E. M. Campbell, Phys. Rev. Lett. 63, 1475 (1989).

${ }^{6}$ Th. Schlegel, S. Bastiani, L. Gremillet, J.-P. Geindre, P. Audebert, J.-C. Gauthier, E. Lefebvre, G. Bonnaud, and J. Delettrez, Phys. Rev. E 60, 2209 (1999)

${ }^{7}$ S. Bastiani, P. Audebert, J. P. Geindre, Th. Schlegel, J. C. Gauthier, C. Quoix, G. Hamoniaux, G. Grillon, and A. Antonetti, Phys. Rev. E 60, 3439 (1999).

${ }^{8}$ M. Kakehata, R. Ueda, H. Takada, K. Torizuka, and M. Obara, Appl. Phys. B: Lasers Opt. B70, S207 (2000).

${ }^{9}$ P. A. VanRompay, M. Nantel, and P. P. Pronko, Appl. Surf. Sci. 127-129, 1023 (1998).

${ }^{10}$ W. L. Kruer, The Physics of Laser Plasma Interactions (Adision-Wesley, New York, 1988); H. A. Baldis, E. M. Campbell, and W. L. Kruer, in Handbook of Plasma Physics, edited by A. Rubenchik and S. Witkowski (North Holland, New York, 1991), Vol. 3, Chap. 9.

${ }^{11}$ P. P. Pronko, Z. Zhang, and P. A. VanRompay, Appl. Surf. Sci. 208-209, 492 (2003).

${ }^{12}$ R. Benattar, J. P. Geindre, P. Audebert, J. C. Gauthier, A. Mysyrowicz, J. P. Chambarret, and A. Antonetti, Opt. Commun. 88, 376 (1992).

${ }^{13}$ D. Duston, R. W. Clark, J. Davis, and J. P. Apruzese, Phys. Rev. A 27, 1441 (1983).

${ }^{14}$ D. T. Attwood, D. W. Sweeny, J. M. Auerbach, and P. H. Y. Lee, Phys. Rev. Lett. 40, 184 (1978).

${ }^{15}$ X. Liu and D. Umstadter, Phys. Rev. Lett. 69, 1935 (1992).

${ }^{16}$ P. P. Pronko, P. A. VanRompay, Z. Zhang, and J. A. Nees, Phys. Rev. Lett. 83, 2596 (1999); P. A. VanRompay, Z. Zhang, J. A. Nees, and P. P. Pronko, Proc. SPIE 3934, 43 (2000). 